\section{Transfusion Related Acute Lung Injury: A severe case triggered with anti-HLA class II antibodies in the recipient}

\author{
HALE BORAZAN • SEBNEM YOSUNKAYA • ALPER YOSUNKAYA
}

$\operatorname{HALE}$ BORAZAN $(\bowtie) \cdot$ ALPER YOSUNKAYA

Department of Anesthesiology and Reanimation

Selcuk University Meram Medical Faculty 42080 Akyokus, Meram Konya, Turkey

Phone: 0903322236550 Fax: 0903322236181 E-mail: borazanh@hotmail.com

SEBNEM YOSUNKAYA Department of Pulmonary Medicine Selcuk University Meram Medical Faculty Konya, Turkey

\begin{abstract}
Transfusion-related acute lung injury (TRALI) is a serious clinical syndrome associated with the transfusion of plasmacontaining blood components. The classic TRALI syndrome is characterized by the suddenly onset of respiratory failure within 2-6 hrs of the transfusion of a blood product, generally transient, resolves within 48-96 hrs spontaneously, and has a better prognosis. Nonetheless there is an expanded definition of TRALI syndrome up to $72 \mathrm{hrs,} \mathrm{which} \mathrm{is} \mathrm{called} \mathrm{delayed}$ TRALI. The potential causes of TRALI can be explained by two distinct mechanism including the anti-leukocyte antibodies in donor plasma or in recipient plasma with the reverse mechanism, and biological response modifiers in susceptible individuals. This report highlights the succesful management of a classic TRALI case that was seen approximately two hours after the transfusion of a packed red blood cell and triggered with anti-HLA class II antibodies in the recipient with reverse mechanism accompanied by neutropenia together.
\end{abstract}

Key words: transfusion, acute lung injury, transfusion related acute lung injury, anti HLA antibodies

\section{Introduction}

Transfusion Related Acute Lung Injury (TRALI), is a clinical syndrome temporally related with transfusion therapy, can include dyspnea, respiratory failure, fever, chills, tachycardia and hypotension. (1) Although it is unlikely, hypertension can be seen with TRALI in some cases. The syndrome can range in severity from a mild dyspneic reaction to acute pulmonary edema with acute respiratory distress syndrome. (2) In a review, an expanded definition of TRALI was varied according to the setting, duration and relation with other risk factors of Acute Lung Injury (ALI) such as classic and delayed TRALI. (3) The classic TRALI syndrome is an uncommon condition characterized by the suddenly onset of respiratory failure within 2-6 hrs of the transfusion of a blood product (3) and there must be no temporal relationship with an alternative risk factor for ALI. (4) It is usually caused by anti-leukocyte antibodies, resolves rapidly, and has a low mortality. However, in the delayed TRALI syndrome the symptoms of TRALI is begun 6-72 hrs after the transfusion and associated with a high mortality. These patients usually have other risk factors for ALI and do not experience the classic definition for TRALI. (3) In cases of TRALI occurring in the setting of transfusion and have an alternative risk factor for ALI, a designation of possible TRALI was added by the Canadian Consensus Conference also. (5) Based on new data, TRALI is now being considered among the three leading causes of transfusion-related morbidity and mortality in recent years. $(6,7)$ The management of not only classic but also delayed TRALI is essentially supportive and most patients having suspected TRALI require mechanical ventilation. (8) Even if the pathophysiology of TRALI can be explained by two distinct mechanism, available evidence strongly suggests that leukocyte antibodies in donor plasma are the predominant mechanism in most cases with antibodies directed at HLA class I antigens in TRALI; however in $5 \%$ to $10 \%$ of the cases, TRALI may develop as a result of antibodies in the recipient reacting with the donor's leukocytes with the reverse mechanism. $(1,9,10)$ TRALI caused by anti HLA class II antibodies without simultaneous presence of anti-HLA class I antibodies have rarely been described.

In this report, we present a case of a classic TRALI syndrome triggered with anti-HLA class II antibodies in the recipient with reverse mechanism accom- 
panied by neutropenia as a result of the administration of packed red blood cell. Therefore we want to define this potentially preventable life-threating condition and to emphasize our experience for its management in the light of the literatures.

\section{Case Report}

A 36 yr-old multiparous (gravity 3, parity 3) woman, operated on for ileus a month ago, had been an operation again for closing ileostomy 2 days ago. Because her operation and postoperative courses were uncomplicated, she was admitted to the surgical ward. On the second postoperative day, one unit of packed red blood cell (PRBC) was planned to correct anemia (Hemoglobin [Hb] $6.2 \mathrm{~g} \mathrm{dl}^{-1}$ ) by the surgeons. Then they started the transfusion in the surgical ward. When she was operated on for ileus, in the preoperative and postoperative period 3 units of PRBC and 2 units of FFP (Fresh Frozen Plasma) which were not leukoreduced were transfused totally in the surgery clinic without any adverse transfusion reactions. Two units of FFP which were transfused to the patient previosly were taken from her brother.

However approximately two hours after the transfusion of the PRBC, the patient complained about difficulty in breathing. Peripheric oxygen saturation $\left(\mathrm{SpO}_{2}\right)$ dropped to $84 \%$ and developed tachypnea. In this condition no erythema, urticaria and hematuria were noticeable. Afterwards, oxygen $\left(\mathrm{O}_{2}\right)$ supplementation was required to maintain adequate oxygenation. At that point she was transferred to the intensive care unit (ICU) urgently with $\mathrm{O}_{2}$ supplementation. Rapid clinical deterioration was noted with a falling $\mathrm{O}_{2}$ saturation (76\%), hypotension (Systolic Blood Pressure $[\mathrm{SBP}]<75 \mathrm{mmHg}$ ), tachycardia (Heart Rate $[\mathrm{HR}]>140$ beat. $\mathrm{min}^{-1}$ ) and tachypnea (frequency $[\mathrm{f}]>35 \mathrm{~min}^{-1}$ ) in the ICU. A chest radiography, taken shortly after the onset of symptoms, showed extensive bilateral pulmonary infiltrates (figure 1). She developed severe respiratory failure $\mathrm{SpO}_{2} 60 \%$, arterial carbon dioxide partial pressure

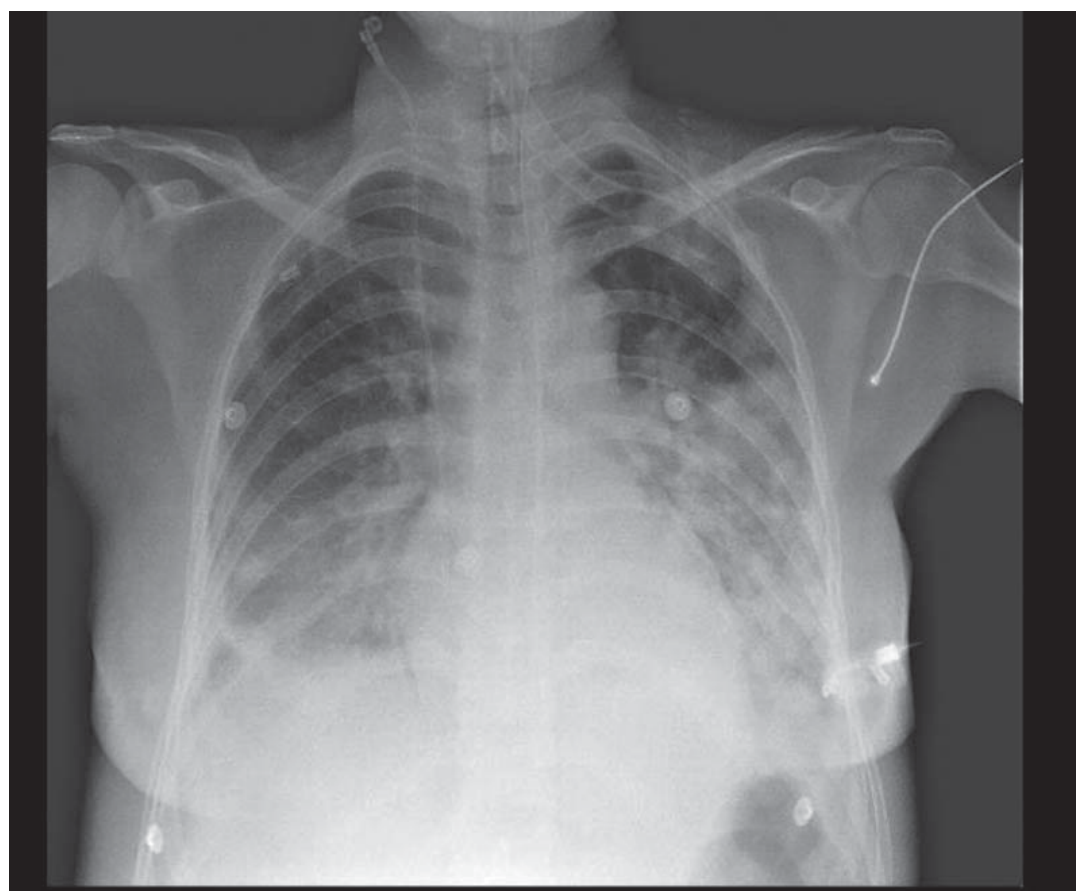

Figure 1. Chest radiography shortly after TRALI development, showing bilateral lung infiltration.

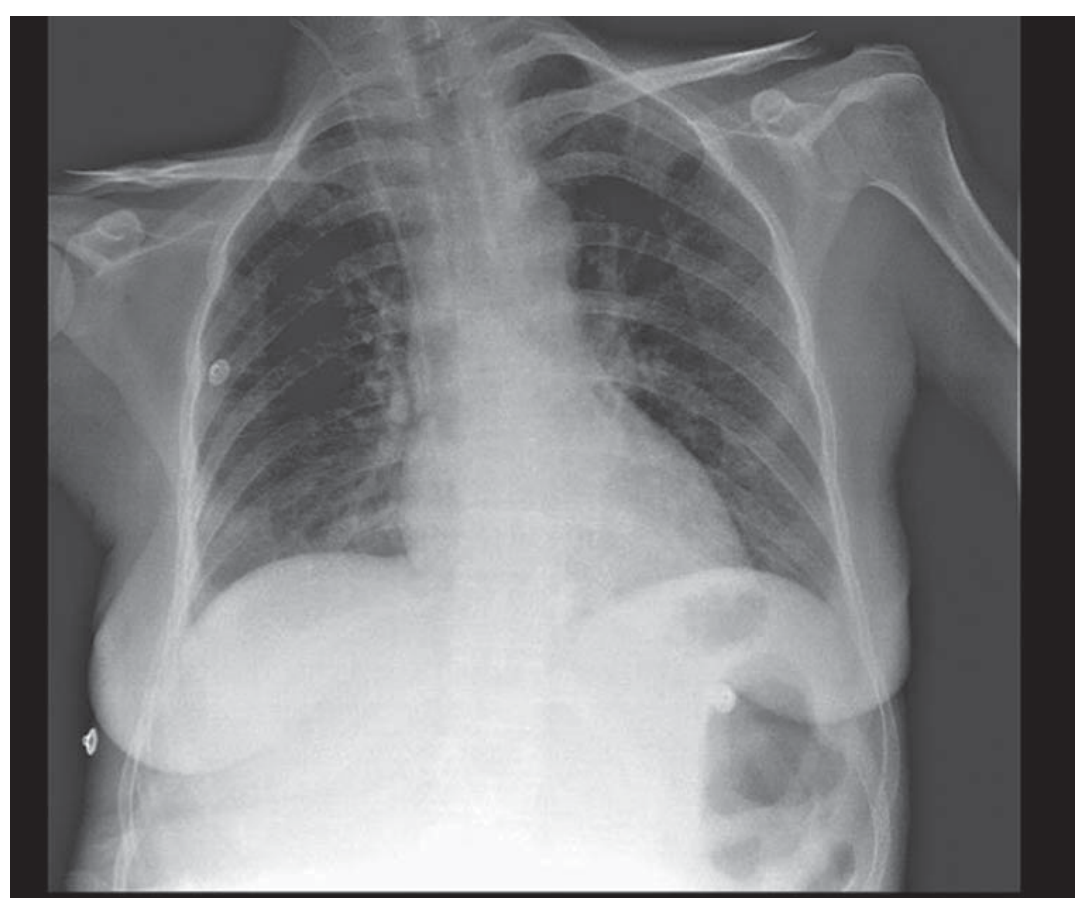

Figure 2. Chest radiography 3 days after the packed red blood cell transfusion.

$\left[\mathrm{PaCO}_{2}\right] 26 \mathrm{mmHg}$, arterial oxygen partial pressure $\left[\mathrm{PaO}_{2}\right] 47.2 \mathrm{mmHg}$, respiratory rate $>40 \mathrm{~min}^{-1}$ ) and hypotension $(\mathrm{SBP}<60 \mathrm{mmHg})$, then intubation and mechanic ventilation (MV) were required to maintain adequate oxygenation. While intubating the patient, the endotracheal tube was filled with large amount of clear, frothy fluid and suctioned repeatedly. She was connected to 
a ventilator (Evita XL, Dräger, Medizintechnix, Lübeck, Germany) in the SIMV (synchronized intermittent mandatory ventilation) mode and ventilated with an oxygen fraction in inspired air $\left(\mathrm{FiO}_{2}\right)$ of $50 \%$ using a Positive end-expiratory pressure (PEEP) of $12 \mathrm{mmHg}$ along with tidal volume of $6 \mathrm{ml} . \mathrm{kg}^{-1}$. While she was mechanically ventilated, the measured plateau pressure was 28 $\mathrm{cmH}_{2} \mathrm{O}$ and she had a clinical ratio of the oxygen partial pressure $\left(\mathrm{PO}_{2}\right)$ to $\mathrm{FiO}_{2}$ of $145 \mathrm{mmHg}$. The hypotension was unresponsive to iv fluid administration. Systolic blood pressure decreased below $60 \mathrm{mmHg}$ necessitating multi-agent hemodynamic support with dopamine, dobutamine and noradrenaline. Though auscultation of the chest revealed rales, cardiac auscultation and electrocardiogram were both normal. While invasive monitoring was initiated, a panel of investigations was ordered immediately. Fluid overload was ruled out by a normal central venous pressure $(\mathrm{CVP}=6 \mathrm{cmH} 2 \mathrm{O})$ and normal echocardiography signs (EF - ejection fraction $=70 \%$ ). Additionally, the patient did not have any clinical signs such as juguler venous distension, systolic hypertension or S3 gallop and angioedema, wheezing, stridor or urticaria. The laboratory findings include leukopenia (White Blood Cell [WBC]: 1.5 K.uL-1), neutropenia (35\%) and monocytopenia (1.1\%), additionally C-reactive protein (CRP) and procalcitonin (PCT) levels were not increased. Re cross-match performed was also negative.

Initial resuscitation procedures comprised MV, hemodynamic support with multi-agents, iv fluids with both crystalloid and colloid and drugs routinely used in our clinic (Asist ${ }^{\circledR} 4 \times 300$ mg,etc). Her arterial SBP increased from $60 \mathrm{mmHg}$ to more than $80 \mathrm{mmHg}$ after approximately 2 hrs. When the WBC count was measured at 4th $\mathrm{hr}$, the leukocyte count (WBC: $7.4 \mathrm{~K} . u L^{-1}$ ), neutrophile (71.1\%), monocyte (5.5\%) was normalized. Over a period of $5 \mathrm{hrs}$, the patient began to respond the symptomatic measures. Her hemodynamic parameters improved and the vasopressor support could be withdrawn after $12 \mathrm{hrs}$.
The result of the HLA typing of posttransfusion blood sample was taken from 2 days after transferring to the laboratory. HLA typing and tests for Panel Reactive Antibody (PRA) were performed by Luminex method (PRA, Luminex, Tepnel, USA). After the transfusion, the patient had multiple anti-HLA antibodies to HLA class II antigens (Anti-DR11, Anti DR12, Anti DR13 and Anti DR16 and \% PRAs of the patient's serum was $66 \%$ ). Based on the clinical signs and laboratory findings including the presence of patient's anti-HLA class II antibodies which potentially could have reacted with donor's leukocyte antigens, the diagnosis of TRALI was become definite.

Recovery from the pulmonary insult was not slower, her chest radiography showed clearance of pulmonary infiltrates in the third day of ventilatory support (figure 2) and weaning was possible in the third day also. She was eventually recovered completely in the next days and discharged from the ICU by the 5th posttransfusion day without any sequel.

\section{Discussion}

Transfusion Related Acute Lung Injury is a complex clinical syndrome, it probably does not represent a simple pathogenic entity. There is increasing evidence that this reaction can be triggered by two distinct mechanisms. (9-11) The subtypes of TRALI based on underlying mechanism into immune (antibody mediated, antiHLA/anti-HNA) or nonimmune (non-antibody mediated, biologically active lipids in stored blood component). (8-10) As shown in our patient, in the immune type TRALI develops as a result of an antigen-antibody reaction.

In $60 \%$ to $80 \%$ of cases, antibodies found in the donor's blood product react with the recipient's leukocytes; however in $5 \%$ to $10 \%$ of the cases, TRALI may develop as a result of antibodies in the recipient reacting with the donor's leukocytes in the reverse mechanism. It was thought that TRALI in our patient can be caused by leukocyte antibodies in the patient's serum against donor WBC antigens. The responsible immunoglobulin antibodies ( $\lg M / G)$ in the patient serum bind and stimulate the small number of circulating leukocytes of the transfused blood have also been described in several studies. $(1,9,10)$ Anti-HLA antibodies do not occur naturally but as a result of immunologic challenge with previous transfusion or pregnancy histories may be the reasons of TRALI in our patient. TRALI has traditionally been associated with the infusion of anti-HLA class I antibodies. However, TRALI caused by anti HLA class II antibodies without simultaneous presence of anti-HLA class I antibodies have rarely been described. In recent report, it has been suggested that anti HLA class II antibodies play a critical role in initiating TRALI development. (12) In our case, it has not been showed the presence of anti-HLA class I antibodies so our case supports the role of anti HLA class II antibodies alone as the cause of TRALI here.

Although the diagnosis of TRALI is primarily clinical and radiographic, (5) it is important to differentiate TRALI clinically from transfusion associated cardiac overload (TACO) and other transfusion reactions like allergic/anaphylactic reactions and bacterial contamination of the transfused blood product. (13) Like TRALI, TACO is increasingly being rediscovered as a common complication of transfusion. TACO is defined by a combination of clinical signs such as tachypnea/respiratory distress, cyanosis, tachycardia, juguler vein distension, post-transfusion hypertension and S3 gallop on cardiac examination. (13) Elevated CVP/PAOP, pulmonary edema with cardiomegaly on chest radiography and prompt response to diuretics are more consistent with TACO. (14) The CVP and wedge pressures are typically low or normal in TRALI but may be elevated if TRALI occurs in the setting of heart failure. $(10,15)$ Therefore, it is important to show the normal ECHO signs and CVP/PAOP (pulmonary artery occlusion pressures) in these patients. In our patient CVP was within normal range $(\mathrm{CVP}=6 \mathrm{cmH} 2 \mathrm{O})$ but we did not measure the wedge pressures. Inserti- 
on of pulmonary artery catheter is not without risk, and because of its limited sensitivity and specificity and also infection risk we did not insert the pulmonary arthery catheter. Moreover, both hypotension and rarely hypertension may be seen in TRALI. $(10,12)$ The hypotension may be unresponsive to iv fluids nevertheless in the presence of normal cardiac function as seen in our patient and she was in need of adminstrating vasopressor support. Also, both production of clear frothy fluid secretions and increased airway pressure have been noted in TRALI and besides two of them were shown in our patient. The $\mathrm{ECHO}$ evaluation of the patient was shown that the left ventricule diameter was $4.9 \mathrm{~cm}$, EF was $70 \%$ and no other heart wall motion defects was denoted also. Though it has been reported that B-type Natriuretic Peptide (BNP) has some value in distinguishing TACO from TRALI, (16) Zhou et al. demonstrated $81 \%$ sensitivity, $89 \%$ specificity, $89 \%$ positive and $81 \%$ negative predictive value and $87 \%$ accuraccy of BNP (B type natriuretic peptide) in diagnosing TACO (16). In any case, we could not measure the level of this hormone in our hospital at that time. But taken together, we thought that clinical manifestations such as normal range of CVP, normal echocardiographic signs (normal LV cavity size and LV ejecion fraction), hypotension and nonappearance of the juguler vein distension, post-transfusion hypertension and S3 gallop on physical examination strongly supports TRALI and help us to exclude of TACO existence in our patient. Since there were no angioedema, wheezing, stridor or urticaria over the neck and trunk, the diagnosis of anaphylactic transfusion reaction was also excluded. Moreover, the res- piratory distress from anaphylactic reaction is related to laryngeal and bronchial edema rather than pulmonary edema so the chest radiograph will generally be clear in anaphylactic reaction to the opposite of TRALI. $(14,17)$ Acute hemoIytic reaction has been excluded based on repeated cross-match results and absence of typical clinical features such as hemoglobinuria or renal failure. (17) The transfusion of contamined blood products may also result in transfusion-related bacterial sepsis that manifests as fever, hypotension and vascular collapse and these patients may also experience ALI. (17) To differentiate this condition, the culturing the component bags and evaluate the WBC, CRP and PCT levels must be assessed also. The coexistence of the leukopenia and absence of not only increased level of CRP and PCT but also hemoglobinuria or renal failure were also chased us from the other possibilities.

However, there are no specific laboratory markers to confirm TRALI, the only routine laboratory parameter that has been associated with TRALI is leukopenia, especially neutropenia. $(7,18)$ It has been an infrequent finding in most of the published cases of TRALI. Nakagawa and Toy (18) found that the patients have a transient, marked decrease in the WBC count where the leukocyte count normalized between 1.5 and 7.5 hours after reaching its nadir. They also noted neutropenia in all cases, even those associated only with anti HLA class II antibodies. This dynamic leukopenia could add support to the diagnosis of TRALI, as it would not be expected to occur in cardiogenic pulmonary edema or TACO. Alike, addition to the other clinical features, leukopenia in our patient speculated the accuracy of our diagnosis because she had neutropenia which was normalized after 4 hrs from the transfusion.

The treatment of both classic and delayed TRALI is primarily supportive and resembles the other forms of ALI. (8) The fundamental part of the treatment is supplemental oxygen. $(13,19)$ Though supportive treatment may be sufficient in mild cases, iv fluids, vasopressor agents and MV may be required in severe TRALI. A low tidal volume, lung protective strategy has been advised for these patients. $(4,19)$ The resolution of TRALI frequently occurs rapidly and most cases present a favorable evoluation without any sequele. $(2,4,13)$ In our case, it was thought that severe TRALI was managed with both iv fluids, vasopressor agents for intractable hypotension and lung protective MV and she was discharged without any sequele.

In conclusion, correct diagnosis and treatment are very important in TRALI. The keys to the diagnosis of TRALI are high clinical suspicion, differentiation the other possibilities of ALI or Acute Respiratory Distress Syndrome (ARDS), radiological, biochemical and immunological findings. Also TRALI can be developed because of antibodies found in the donor's blood product, it is necessary to know that it may develop as a result of antibodies in the recipient reacting with the donor's leukocytes in the reverse mechanism. We thought that, in the cases which TRALI associated only with anti HLA class II antibodies, it is important to minimize the risks for infection becuse of the relationship between anti HLA class II antibodies and leukopenia, which might be associated with patient and intensive care factors especially device related infections. 


\section{REFERENCES}

1. Kopko PM, Holland PV. Transfusion-related acute lung injury. Br J Haematol 1999;105:322-9.

2. Kopko PM, Marshall CS, MacKenzie MR, Holland PV, Popovsky MA. Transfusion-related acute lung injury: report of a clinical look-back investigation. JAMA 2002;287:1968-71.

3. Marik PE, Corwin HL. Acute lung injury following blood transfusion: Expanding the definition. Crit Care Med 2008;36:3080-84.

4. Toy P, Popovsky MA, Abraham E, Ambrusso DR, Holnes LG, Kopko PM, et al. The National Heart Lung and Blood Institute Working Group on TRALI. Transfusion-related acute lung injury: Definition and review. Crit Care Med 2005;33:721-6.

5. Kleinman S, Caulfield T, Chan P, Davenport R, McFarland J, McPhedran S, et al. Toward on understanding of transfusion-related acute lung injury: statement of a consensus panel. Transfusion 2004;44:1774-89.

6. Bux J. Transfusion-related acute lung injury (TRALI): a serious adverse event of blood transfusion. Vox Sang 2005;89(1):1-10.

7. Sachs UJ, Kauschat D, Bein G. White blood cell-reactive antibodies are undetectable in solvent/detergent plasma. Transfusion. 2005;45(10):1628-31.

8. Gajic O, Rana R, Winters JL, YIImaz M, Mendez JL, Rickman OB, et al. Transfusion related acute lung injury in the critically ill: prospective nested case-control study. Am J Respir Crit Care Med 2007;176:886-91.

9. Wallis J. Transfusion-related acute lung injury (TRALI) — under-diagnosed and under-reported. Br J Anaesth 2003;90:573-6.

10. Jawa RS, Anillo S, Kulaylat MN. Transfusion-related acute lung injury. J Intensive Care Med 2008;23:109-21.

11. Holnes L, Knippen MA, Simmons L, Lachenbruch PA. Fatalities caused by TRALI. Transfus Med Rev 2004;18:184-8.

12. Nishimura M, Hashimoto S, Takanashi M, Okazaki H, Satake M, Nakajima K. Role of anti-human leucocyte antigen class II alloantibody and monocytes in development of transfusion-related acute lung injury. Transfus Med 2007;17:129-34.

13. Cherry T, Steciuk M, Reddy VVB, Marques MB. Transfusion-Related Acute Lung Injury. Am J Clin Pathol 2008;129:287-97.

14. Benson AB, Mors M, Silliman CC. Transfusion-related acute lung injury (TRALI): a clinical review with emphasis on the critically ill. Br $J$ Haematol 2009;147:431-43.

15. Webert KE, Blajchman MA. Transfusion-related acute lung injury. Curr Opin Hematol 2005;12:480-7.

16. Zhou L, Giacherio D, Cooling L, Davenport RD. Use of B-natriuretic peptide as a diagnostic marker in the differential diagnosis of transfusion-associated circulatory overload. Transfusion. 2005;45:1056-63.

17. Silliman CC, McLaughlin NJ. Transfusion-related acute lung injury. Blood Reviews 2006;20:139-59.

18. Nakagawa M, Toy P. Acute and transient decrease in neutrophil count in transfusion-related acute lung injury: cases at one hospital. Transfusion 2004;44:1689-94.

19. Kalhan R, Mikkelsen M, Dedhiya P, Christie J, Gaughan C, Lanken PN, et al. Underuse of lung protective ventilation: analysis of potential factors to explain physician behavior. Crit Care Med 2006;34:300-6.

It was presented in 18th International Intensive Care Symposium as a poster 